BULLETIN (New Series) OF THE

AMERICAN MATHEMATICAL SOCIETY

Volume 50, Number 4, October 2013, Pages 691-696

S 0273-0979(2013)01418-6

Article electronically published on May 1, 2013

Lie superalgebras and enveloping algebras, by Ian Musson, Graduate Studies in

Mathematics, Vol. 131, American Mathematical Society, Providence, RI, 2012,

xx+488 pp., hardcover, \$87.00, ISBN 978-0-8128-6867-6

\title{
BACKGROUND AND QUESTIONS
}

The history of Lie superalgebras starts in mid-1970s. The original motivation for their study comes from physics as a way towards understanding the mathematical foundation of supersymmetry.

A Lie superalgebra $\mathfrak{g}$ is a $\mathbb{Z}_{2}$-graded vector space $\mathfrak{g}_{0} \oplus \mathfrak{g}_{1}$ with a bracket $[\cdot, \cdot]$ satisfying $\mathbb{Z}_{2}$-graded versions of skew symmety and Jacobi identity

$$
\begin{gathered}
{[x, y]=-(-1)^{p(x) p(y)}[y, x],} \\
{[x,[y, z]]=[[x, y], z]+(-1)^{p(x) p(y)}[y,[x, z]],}
\end{gathered}
$$

where $p(x)$ is the parity of $x$ for a homogeneous $x \in \mathfrak{g}$. One can see that $\mathfrak{g}_{0}$ is a Lie algebra and $\mathfrak{g}_{1}$ is a $\mathfrak{g}_{0}$-module.

The first example is the Lie superalgebra $g l(m \mid n)$ realized as the algebra of endomorphisms of a $\mathbb{Z}_{2}$-graded vector space $V$ with $\operatorname{dim} V_{0}=m, \operatorname{dim} V_{1}=n$. The bracket is defined by

$$
[X, Y]=X Y-(-1)^{p(X) p(Y)} Y X
$$

on homogeneous elements and is extended on the whole algebra by linearity. If one defines the supertrace by the formula

$$
\operatorname{str} X=\operatorname{tr}_{V_{0}} X-\operatorname{tr}_{V_{1}} X
$$

then $\operatorname{str}[X, Y]=0$ and hence the set of traceless matrices $\operatorname{sl}(m \mid n)$ is again a Lie superalgebra. This one is already simple unless $m \neq n$. But if $m=n$, the supertrace of a scalar matrix is zero. Hence $\operatorname{sl}(n \mid n)$ has a nontrivial center, and it is not hard to see that this center does not split as an ideal. The reader acquainted with Lie algebras in positive characteristic can see immediately the analogy with this situation. This analogy goes much further. Indeed the famous Weyl theorem (claiming that any finite-dimensional representation of a simple Lie algebra over a field of characteristic zero is completely reducible) is not true for simple Lie superalgebras, just as in the positive characteristic setting.

In 1977 Kac published the classification of finite-dimensional simple Lie superalgebras over an algebraically closed field of characteristic zero; see [14. Not all simple Lie superalgebras have an invariant quadratic form. It is also not true that the even part of a simple Lie superalgebra is always reductive. Simple Lie superalgebras, which satisfy both aforementioned conditions, are called basic classical. They can be defined in terms of Cartan matrices and Chevalley generators and have root decompositions and Dynkin diagrams. One can try to generalize the classical structure theory to these superalgebras. In this way one encounters a new phenomenon: existence of several nonisomorphic Dynkin diagrams defining isomorphic superalgebras. In other words, there are usually several nonconjugate Borel subalgebras. For instance take $\mathfrak{g}=\operatorname{sl}(1 \mid 2)$ acting on the superspace $\mathbb{C}^{1 / 2}$. The Borel

2000 Mathematics Subject Classification. Primary 17B35, 17B10, 17B05.

(C) 2013 American Mathematical Society Reverts to public domain 28 years from publication 
subalgebra preserving the flag $\mathbb{C}^{1 \mid 0} \subset \mathbb{C}^{1 \mid 1}$ is not conjugate to the one preserving the flag $\mathbb{C}^{0 \mid 1} \subset \mathbb{C}^{0 \mid 2}$. Those can be connected by so-called odd reflection and that leads to a nontrivial generalization of the Weyl group in the super case, namely a groupoid [22].

The classification of finite-dimensional irreducible representations over basic classical simple Lie superalgebras can be done in terms of highest weights. It is contained in the original Kac paper [14. In [15] Kac gave a superanalogue of the Weyl character formula for generic weights called typical. In general, the problem of calculating irreducible characters remained open for more than 25 years and there is a tremendous amount of literature on this subject, starting with [1. We do not give a complete list due to lack of space. Let us just say that irreducible modules with nontypical highest weights have smaller characters than expected.

The beauty of Kac classification, as well as a wide range of difficult and interesting problems, drew the attention of mathematicians. So people began to study Lie superalgebras and their representations independently of the original application to physics.

For the last 35 years substantial progress was made, most notably obtaining characters of irreducible representations and discovering superanalogues of SchurWeyl duality.

Let us start by explaining two versions of Schur-Weyl duality in the supercase. Both were discovered by A. Sergeev [24]. Let $V$ be the standard representation of $g l(m \mid n)$. The $d$ th tensor power $V^{\otimes d}$ is again a representation of $g l(m \mid n)$. On the other hand, there is a natural action of the symmetric group $S_{d}$ on the same space. It was proven by Sergeev that $g l(m \mid n)$ and $S_{d}$ form a dual pair and $V^{\otimes d}$ has a decomposition

$$
V^{\otimes d}=\bigoplus V(\lambda) \otimes Y(\lambda),
$$

where summation is taken over all Young diagrams with $d$ boxes which do not contain the forbidden box with coordinates $(n+1, m+1), Y(\lambda)$ is the irreducible representation of $S_{d}$ associated with $\lambda$, and $V(\lambda)$ is an irreducible representation of $g l(m \mid n)$. The classical case $n=0$ and the condition of the forbidden box translates into the condition that the number of rows in $\lambda$ is less than $n+1$. This approach leads to the theory of supersymmetric polynomials, superanalogues of Schur polynomials, and character formulas for $V(\lambda)$. However, plenty of finite-dimensional irreducible representations of $g l(m \mid n)$ cannot be obtained in this way.

It is worth mentioning the superanalogue of Schur-Weyl duality for the Lie superalgebra $q(n)$. The latter is defined as a subalgebra of $g l(n \mid n)$ consisting of matrices of the form $\left(\begin{array}{c}A B \\ B A\end{array}\right)$ for some $n \times n$ matrices $A$ and $B$. If $V$ is the $n \mid n$-dimensional representation of $q(n)$, the centralizer $B(d)$ of $q(n)$ in $V^{\otimes d}$ is a semisimple algebra containing $S_{d}$. Finite-dimensional irreducible representations of $B(d)$ are the same as projective representations of $S_{d}$. Thus, polynomial irreducible representations of $q(n)$ are in duality with projective representations of symmetric groups; see [25].

While the duality method can be used to calculate characters of some finitedimensional representations of $\operatorname{sl}(m \mid n)$ and $q(n)$ and indeed leads to beautiful character formulas, the method has its limits and cannot be applied to all representations. To obtain all irreducible characters, one should understand the structure of the representation in the space of mixed tensors $T^{p, q}=V^{\otimes p} \otimes\left(V^{*}\right)^{\otimes q}$. Unfortunately, $T^{p, q}$ is not completely reducible and the centralizer of $\operatorname{sl}(m \mid n)$ in $T^{p, q}$ is not semisimple. Quite recently in [8] it was proven that it is a quotient of the 
so-called walled Brauer algebra and indecomposable components of $T^{p, q}$ were classified. From this point of view one can realize the category of tensor representations of $s l(m \mid n)$ as a quotient of the Deligne category $S L(t)$-modules with specialization $t=m-n$.

The problem of finding the character of all irreducible finite-dimensional representations was solved originally for $\operatorname{sl}(m \mid n)$ in [23] using the superanalogue of the Borel-Weil-Bott approach. The idea is to realize irreducible representations geometrically via cohomology of line bundles over flag supermanifolds. Again the reader familiar with the positive characteristic case can see the analogy with Weyl modules for Chevalley groups. This approach is based on many important results in supergeometry obtained in the 1980s, in particular on the result of Penkov 19] that the Borel-Weil-Bott theorem holds for line bundles with typical weights. This approach was applied successfully for $q(n)$ in [20] and to the orthosymplectic superalgebra in [13].

In 2003 Brundan in 2 discovered another way to deal with finite-dimensional representations of $\operatorname{sl}(m \mid n)$ using the categorification method. He identified the Grothendieck group of finite-dimensional $s l(m \mid n)$-modules with a specific tensor representation of $s l(\infty)$ and realized the action of the Chevalley generators of the latter by very naturally defined translation functors. In this way, he was able to solve the problem by reducing to combinatorics of canonical bases for $s l(\infty)$. He applied the same method for $q(n)$ in [3].

After the remarkable paper of Brundan, the fast developing categorification approach connected representation theory of Lie superalgebras with other branches of representation theory, such as representation theory, of infinite Lie algebras and quantum groups. Let us just list some examples. In [4] the language of Khovanov diagram algebras was used to construct the algebra of endomorphisms of the projective generator of the category of finite-dimensional representations of $\operatorname{sl}(m \mid n)$. In [5] those results were used to reveal the relationship with Deligne categories and walled Brauer algebras. On the other hand, Brundan's approach can be used for calculating the characters of infinite-dimensional irreducible highest weight representations in terms of Kazhdan-Lusztig polynomials for category $\mathcal{O}$. It was done recently in [7] for $\operatorname{sl}(m \mid n)$; see also [6].

As in the classical case, simple finite-dimensional Lie superalgebras have infinitedimensional generalizations: affine Lie superalgebras. Finding character formulas of irreducible highest weight modules of affine Lie superalgebras is a difficult problem, which remains open in the general case. For certain highest weights, however, a beautiful formula was conjectured by Kac and Wakimoto in [16. In the case of the trivial module this formula gives the so-called Weyl denominator identity recently proved by M. Gorelik; see [10, 11].

On the other hand, denominator identities for affine Lie superalgebras are interesting due to their connections with certain identities in number theory; see [16].

These latest developments caused a growth of interest in Lie superalgebras in the last decade. However, with the exception of [21] where the details of Kac classification are explained, there were no monographs on the theory of Lie superalgebras.

The book under review happily fills this gap. It contains all basic results concerning algebraic aspects of Lie superalgebras and is an excellent introduction to the field. The reader does not need much background. Basic ring theory and structure theory of Lie algebras are sufficient for understanding. 


\section{DESCRIPTION OF THE CONTENTS OF THE BOOK}

In the first five chapters of the book, a detailed description of classical and exceptional Lie superalgebras is given together with the classification of Borel subalgebras, Dynkin diagrams, and groups of automorphisms. In Chapter 5 the notion of a contragredient Lie superalgebra is introduced. It gives a unified approach to the results of the previous chapters and to some extent to infinite-dimensional Lie superalgebras.

Chapters 6 through 10 and 13 of the book deal with ring theoretical properties of universal enveloping algebras of Lie superalgebras. Note that the essential difference with the Lie algebra case is caused by the fact that universal enveloping superalgebras have zero divisors. To see that, consider a superalgebra $\mathfrak{g}$ with zero bracket. Then its universal enveloping algebra is isomorphic to $S\left(\mathfrak{g}_{0}\right) \otimes \Lambda\left(\mathfrak{g}_{1}\right)$ and hence has zero divisors as soon as $\mathfrak{g}_{1} \neq 0$. While the fundamental results, such as the Poincare-Birkhoff-Witt theorem and Witt's theorem, have their analogues for superalgebras, further analogy stops working very quickly. A good example is the center $Z(\mathfrak{g})$ of the universal enveloping algebra $U(\mathfrak{g})$ of a simple Lie superalgebra $\mathfrak{g}$. In contrast with the classical case, $Z(\mathfrak{g})$ in most cases is not Noetherian. Let us recall that for a semisimple Lie algebra the fibers of the natural map from the primitive spectrum of $U(\mathfrak{g})$ to the maximal spectrum Specm of $Z(\mathfrak{g})$ has finite fibers with transitive action of the Weyl group on each fiber. Complete classification of primitive ideals can be done therefore in terms of the Weyl group. For a semisimple superalgebra, fibers are finite only over generic points of Specm $Z(\mathfrak{g})$. The question of the classification of primitive ideals is open in almost all cases; for some results see [17, [18.

In the book under review universal enveloping superalgebras are treated in the spirit of Diximier's book on enveloping algebras [9]. That includes a substantial amount of material on the category of highest weight modules, Shapovalov's determinant, and Jantzen filtration. The results concerning primitive ideals and modules are collected in Chapters 15 and 17, most of them are obtained by the author of the book. Chapter 16 contains a nice review of cohomology theory of Lie superalgebras from the algebraic point of view. For a geometric approach to this topic see 12 .

Finite-dimensional representations of simple Lie superalgebras are treated in two different ways. Polynomial representations of $g l(m, n)$ are constructed via SergeevSchur-Weyl duality, and character formulas are obtained using combinatorics of Young tableaux. For arbitrary simple Lie superalgebras all finite-dimensional irreducible representations are constructed via the highest weight theory culminating in the Kac-Weyl formula for the character of a typical irreducible representation.

The last chapter of the book includes results of Gorelik, Kac, and Wakimoto generalizing character formulas for affine Lie superalgebras. These formulas have realization via combinatorial identities appearing in number theory.

The book contains many interesting examples and exercises together with useful hints, as well as an appendix on some ring theory, Hopf algebras, and combinatorics. It contains a clear description of the basic tools of the subject together with a solid construction of the foundations of the theory (Kac classification, Sergeev-SchurWeyl duality, cohomology theory etc.... ). Moreover, the main point of view is not lost in technicalities. It is an excellent reference text for a graduate course, since it contains clearly written detailed proofs. As mentioned before, there was a real 
need for such a book. It is surely very useful for a beginner or anyone who wants to enter current research on the topic.

\section{REFERENCES}

[1] I. N. Bernšteĭn and D. A. Leĭtes, A formula for the characters of the irreducible finitedimensional representations of Lie superalgebras of series Gl and sl, C. R. Acad. Bulgare Sci. 33 (1980), no. 8, 1049-1051 (Russian). MR620836 (82j:17020a)

[2] Jonathan Brundan, Kazhdan-Lusztig polynomials and character formulae for the Lie superalgebra $\mathfrak{g l}(m \mid n)$, J. Amer. Math. Soc. 16 (2003), no. 1, 185-231, DOI 10.1090/S0894-034702-00408-3. MR.1937204 (2003k:17007)

[3] Jonathan Brundan, Kazhdan-Lusztig polynomials and character formulae for the Lie superalgebra $\mathfrak{q}(n)$, Adv. Math. 182 (2004), no. 1, 28-77, DOI 10.1016/S0001-8708(03)00073-2. MR2028496 (2004m:17018)

[4] Jonathan Brundan and Catharina Stroppel, Highest weight categories arising from Khovanov's diagram algebra IV: the general linear supergroup, J. Eur. Math. Soc. (JEMS) 14 (2012), no. 2, 373-419, DOI 10.4171/JEMS/306. MR2881300(2012m:17009)

[5] Jonathan Brundan and Catharina Stroppel, Gradings on walled Brauer algebras and Khovanov's arc algebra, Adv. Math. 231 (2012), no. 2, 709-773, DOI 10.1016/j.aim.2012.05.016. MR2955190

[6] Shun-Jen Cheng, Ngau Lam, and Weiqiang Wang, Super duality and irreducible characters of ortho-symplectic Lie superalgebras, Invent. Math. 183 (2011), no. 1, 189-224, DOI 10.1007/s00222-010-0277-4. MR2755062 (2012f:17011)

[7] S. J. Cheng, N. Lam, W. Wang, Brundan-Kazhdan-Lusztig conjecture for general Lie superalgebras. arXiv:1203.0092v3.

[8] Jonathan Comes and Benjamin Wilson, Deligne's category $\operatorname{Rep}\left(G L_{\delta}\right)$ and representations of general linear supergroups, Represent. Theory 16 (2012), 568-609, DOI 10.1090/S1088-41652012-00425-3. MR2998810

[9] Jacques Dixmier, Enveloping algebras, Graduate Studies in Mathematics, vol. 11, American Mathematical Society, Providence, RI, 1996. Revised reprint of the 1977 translation. MR:1393197 (97c:17010)

[10] Maria Gorelik, Weyl denominator identity for affine Lie superalgebras with non-zero dual Coxeter number, J. Algebra 337 (2011), 50-62, DOI 10.1016/j.jalgebra.2011.04.011. MR:2796063 (2012c:17038)

[11] Maria Gorelik and Shifra Reif, A denominator identity for affine Lie superalgebras with zero dual Coxeter number, Algebra Number Theory 6 (2012), no. 5, 1043-1059, DOI 10.2140/ant.2012.6.1043. MR2968633

[12] Caroline Gruson, Finitude de l'homologie de certains modules de dimension finie sur une super algèbre de Lie, Ann. Inst. Fourier (Grenoble) 47 (1997), no. 2, 531-553 (French, with English summary). MR 1450424 (98b:17024)

[13] Caroline Gruson and Vera Serganova, Cohomology of generalized supergrassmannians and character formulae for basic classical Lie superalgebras, Proc. Lond. Math. Soc. (3) 101 (2010), no. 3, 852-892, DOI 10.1112/plms/pdq014. MR2734963 (2012a:17010)

[14] V. G. Kac, Lie superalgebras, Advances in Math. 26 (1977), no. 1, 8-96. MR.0486011 (58 \#5803)

[15] V. G. Kac, Characters of typical representations of classical Lie superalgebras, Comm. Algebra 5 (1977), no. 8, 889-897. MR.0444725 (56 \#3075)

[16] Victor G. Kac and Minoru Wakimoto, Integrable highest weight modules over affine superalgebras and number theory, Lie theory and geometry, Progr. Math., vol. 123, Birkhäuser Boston, Boston, MA, 1994, pp. 415-456. MR1327543 (96j:11056)

[17] Ian M. Musson, A classification of primitive ideals in the enveloping algebra of a classical simple Lie superalgebra, Adv. Math. 91 (1992), no. 2, 252-268, DOI 10.1016/0001-8708(92)90018G. MR 1149625 (93c:17022)

[18] Ian M. Musson, Enveloping algebras of Lie superalgebras: a survey, Azumaya algebras, actions, and modules (Bloomington, IN, 1990), Contemp. Math., vol. 124, Amer. Math. Soc., Providence, RI, 1992, pp. 141-149, DOI 10.1090/conm/124/1144033. MR 1144033 (93b:17012) 
[19] I. B. Penkov, Borel-Weil-Bott theory for classical Lie supergroups, Current problems in mathematics. Newest results, Vol. 32, Itogi Nauki i Tekhniki, Akad. Nauk SSSR Vsesoyuz. Inst. Nauchn. i Tekhn. Inform., Moscow, 1988, pp. 71-124 (Russian). Translated in J. Soviet Math. 51 (1990), no. 1, 2108-2140. MR.957752 (90f:22018)

[20] I. Penkov and V. Serganova, Characters of irreducible $G$-modules and cohomology of $G / P$ for the Lie supergroup $G=Q(N)$, J. Math. Sci. (New York) 84 (1997), no. 5, 1382-1412, DOI 10.1007/BF02399196. Algebraic geometry, 7. MR1465520 (98i:17010)

[21] Manfred Scheunert, The theory of Lie superalgebras. An introduction, Lecture Notes in Mathematics, vol. 716, Springer, Berlin, 1979. MR537441(80i:17005)

[22] Vera Serganova, Kac-Moody superalgebras and integrability, Developments and trends in infinite-dimensional Lie theory, Progr. Math., vol. 288, Birkhäuser Boston Inc., Boston, MA, 2011, pp. 169-218, DOI 10.1007/978-0-8176-4741-4_6. MR2743764(2011m:17056)

[23] Vera Serganova, Kazhdan-Lusztig polynomials and character formula for the Lie superalgebra $\mathfrak{g l}(m \mid n)$, Selecta Math. (N.S.) 2 (1996), no. 4, 607-651, DOI 10.1007/PL00001385. MR.1443186(98f:17007)

[24] A. N. Sergeev, Representations of the Lie superalgebras $\mathfrak{g l}(n, m)$ and $Q(n)$ in a space of tensors, Funktsional. Anal. i Prilozhen. 18 (1984), no. 1, 80-81 (Russian). MR739101 (86b:17005)

[25] Alexander Sergeev, The Howe duality and the projective representations of symmetric groups, Represent. Theory 3 (1999), 416-434 (electronic), DOI 10.1090/S1088-4165-99-000850. MR1722115 (2000j:20021)

Vera Serganova

University of CAlifornia, Berkeley E-mail address: serganov@math.berkeley.edu 